\title{
Synthesis of some azetidonone and 1, 3-oxazepine deritives from thymol
}

\author{
A. kh. Ahmad Linda R. Butti \\ Department of chemistry / College of Education \\ Mosul University
}

Received

14 / $11 / 2011$
Accepted

27 / 01 / 2013

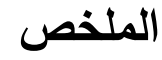

في هذا البحث تم تحضير بعض مركبات أزنتين-2-اون (16-11) من تفاعل مشتقات

الهيدرازون (10-3) مع كلورو استيل الكلورايد بوجود ثلاثي اثيل أمين، حيث الهيدرازونات نم

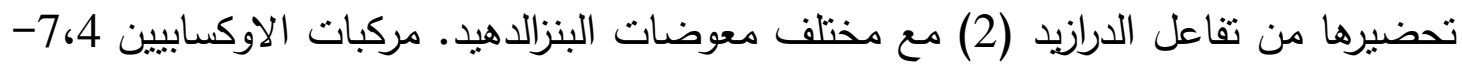

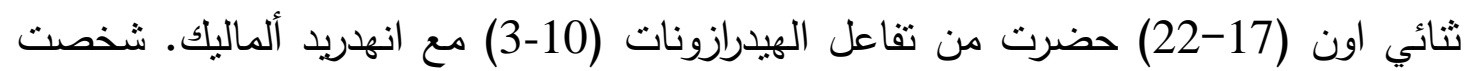
المركبات الناتجة بالطرق الطيفية والفيزياويه المتاحة.
\end{abstract}

\begin{abstract}
A series of some azetidines -2-one derivative (11-16) have been synthesized by cyclocondensation of various hydrazones derivatives (310) with chloroacetyl chloride in the presence of triethylamine. Hydrazones (3-10) were synthesized from the reaction of hydrazide (2) with various substituted benzaldehyde. Oxazepain 4,7-dion derivatives (17-22) were synthesized from the reaction of hydrazones derivatives (310) with maleic anhydride
\end{abstract}

\section{INTRODUCTION}

Azetidine-2-one and 4, 7-oxazepaine derivatives were reported to posse's antibacterial, antifungal ${ }^{(1-3)}$, antianflammatory and antitubercular activities $^{(4)}$ also oxazepine derivatives used as neuroleptic and as antidepressant $^{(5,6)}$

Azetidine-2-one can be prepared from ketene-imines cycloaddition $^{(7)}$ reaction, although many synthetic methods have been developed, Bhat and etal. ${ }^{(8)}$ synthesized Schiff's bases from condensation of acid hydrazine of p-anisidine with aromatic aldehydes, which on treatment with chloroacetylchloride in the presences of triethylamine afforded 2-azetidiones.1, 4-benzoxazepine-2, 5- $(1 \mathrm{H}, 3 \mathrm{H})$-dione was 
prepared from the reaction of o.aminobenzoic acid with choroacetyl chloride. Alkyl substituted derivatives were prepared from the reaction of the corresponding alkyl halide with benzoxazepinedione in the presence of a suspension of sodium hydride in dimethylforamide. ${ }^{(9)}$

In this paper we report the synthesis of some azetidinone and oxazepine derived from ethyl thymoxy acetate

\section{EXPERIMENTAL}

Melting points were measured on akofler hot stage. The IR spectra were recorded by using infra red spectrophotometer model Tensor 27 Bruker co. Germany. The ${ }^{1} \mathrm{HNMR}$ were recorded by Bruker ultra shield Dp"400 MHz Avancell (2008), Ortaduteknek university using $\mathrm{CDCL}_{3}$ as solvent with tetra methylsaline as references.

\section{Ethyl thymoxy acetate (1)}

This compound was prepared from the reaction of (0.06 mole, $9.0 \mathrm{~g})$ thymol, $(0.06 \mathrm{~mole}, 8.28 \mathrm{~g})$ anhydrous potassium carbonate and $(0.06 \mathrm{~mole}$, $10.14 \mathrm{~g}$ ) bromoethylacetate following the method describe in the literature $^{(10)}$, gave $96 \%$, colorless oily product.

\section{Themoxy acetic acid hydrazide (2)}

This compound was prepared from the reaction of $(0.05 \mathrm{~mole}$, $12.89 \mathrm{~g})$ ester (1) and $(0.25$ mole, $12.5 \mathrm{~g})$ hydrazine hydrate $99 \%$ as mentioned in the literature ${ }^{(10)}$, yield $87 \%$, m.p. $\left(93-95^{\circ} \mathrm{C}\right)$, lit. $\left(93-95^{\circ} \mathrm{C}\right)$

\section{Hydrazones $^{(11)}$ (3-10)}

A mixture of hydrazide (3) $(2.22 \mathrm{~g}, 0.01 \mathrm{~mole})$ in $25 \mathrm{ml}$ ethanol, and substituted aromatic aldehyde $(0.01 \mathrm{~mole})$ in $25 \mathrm{ml}$ ethanol was added. The reaction mixture was heated under reflux for 2 hours after completion of reaction; the reaction mixture was allowed to cool. The precipitate was filtered and recrystallized from ethanol, to give the hydrazones (3-10). Some physical and spectral data indicated in tables $(1,4)$.

\section{Substituted azetidine-2-one (11-16)}

General procedure for synthesis ${ }^{(12)}$

A solution of hydrazones derivatives(3-10) (0.005mole) and triethylamine $(0.01 \mathrm{~mole})$ in $40 \mathrm{ml} 1,4$-dioxane, Chloroacetyl chloride (0.01 moles) was added as drop wise with stirring at room temperature for 20 minutes, and then the mixture was refluxed for 3hours. The reaction mixture concentrated then poured into ice-water and titled compounds were isolated by ethyl acetate, dried and recrystallized from absolute ethanol, yield the required products(11-16). The physical and spectral data were listed in tables $(2,5)$. 
3- Thymoxy methyl acetamido-2-aryl -2, 3-dihydro 1, 3-oxazepine, 4, 7-dione ${ }^{(12)}(17-22)$

A mixture of hydrazone derivatives $(3-7,9)$ in $30 \mathrm{ml}$ dry benzene and maleic anhydride $(0.29 \mathrm{~g}, 0.003$ moles $)$ were refluxed for 2 hours, the solvent was evaporated, and precipitate was recrystallized from ethanol, giving the required products. The physical and spectral data were listed in table $(3,6)$.

\section{RESULTS AND DISCUSSION}

The hydrazides (2) were obtained from refluxing ester (1) with 99\% hydrazine hydrate in absolute ethanol. These hydrazides were identified by IR which exhibits characteristic peak at $\left(3316 \mathrm{~cm}^{-1}\right)$ for the $(\mathrm{N}-\mathrm{H})$ stretching, peak at for the carboxyl group appear at $\left(1678 \mathrm{~cm}^{-1}\right)$ which lower than the carbonyl ester $\left(1739 \mathrm{~cm}^{-1}\right)$ due to the presences of resonances effect ${ }^{(13)}$

Hydrazones (3-10) were prepared by reaction of the thymoxy acetic acid hydrazide (2) and different aryl aldehyde. The structure of hydrazones were elucidated from spectra evidence, peak at (1688-1697 $\left.\mathrm{cm}^{-1}\right)$ for the carbonyl group, also the peak at $\left(1604-1614 \mathrm{~cm}^{-1}\right)$ for $\mathrm{C}=\mathrm{N}$. In addition to that the stretching banding at $\left(3317-3487 \mathrm{~cm}^{-1}\right)$ is assigned for N-H. The IR spectral data shows at table (4).

The reaction product of hydrazones derivatives (3-10) with chloroacetylchloride elucidated from IR and ${ }^{1} \mathrm{HNMR}$. The IR shows the absence of stretching bands at (1604-1614 $\left.\mathrm{cm}^{-1}\right)$ for $\mathrm{C}=\mathrm{N}$ group and the bands at (1696-1733 $\left.\mathrm{cm}^{-1}\right)$ for the carbonyl lactam stretching and banding at $\left(1422-1433 \mathrm{~cm}^{-1}\right)$ for $\mathrm{C}-\mathrm{N}$, while absorbing bands at $\left(1503-1508 \mathrm{~cm}^{-1}\right)$ for $\mathrm{C}=\mathrm{C}$ in addition to that bands at $\left(711-762 \mathrm{~cm}^{-1}\right)$ for $\mathrm{C}-\mathrm{CL}$. The IR as shows in table (5).

The ${ }^{1} \mathrm{HNMR}$ spectrum for compounds (16) shows bands as multiple at $1.245 \mathrm{ppm}$ for $\mathrm{CH}\left(\mathrm{CH}_{3}\right)_{2}$, singlet band at $2.314 \mathrm{ppm}$ for Ph$\mathrm{CH}_{3}$, multiple band at $3.272 \mathrm{ppm}$ for $\mathrm{CH}<$, singlet band at $4.132 \mathrm{ppm}$ for $\mathrm{CH}_{2}$, doublet band for CHCL at 4.613 also singlet band at $5.134 \mathrm{ppm}$ for $\mathrm{CH}$, multiple bands at 6.602-8.137 ppm for Ar-H finally band at 9.049 ppm for N-H, the ${ }^{1} \mathrm{HNMR}$ for compound (16) table (5).

Refluxing hydrazones (3-10) with maleic anhydride will produce oxazepine -4,7-dione derivatives (17-22) and their structure was confirmed by spectroscopic data. IR shows the carbonyl lactones at $\left(1681-1700 \mathrm{~cm}^{-1}\right)$ and carbonyl amide at $\left(1603-1639 \mathrm{~cm}^{-1}\right)$ other absorption bands shows in table (6). The ${ }^{1} \mathrm{HNMR}$ spectrum for compound (18) showed results that confirm our expectation as mention in table (6).

Table (1): Some physical constant for compounds (3-10)

\begin{tabular}{|c|c|c|c|c|}
\hline Comp. No. & $\mathrm{R}$ & M.p. $\left(\mathrm{C}^{\circ}\right)$ & Yield(\%) & Color \\
\hline 3 & $4-\mathrm{OH}$ & $111-113$ & 85 & yellow \\
\hline 4 & $4-\mathrm{CL}$ & $165-167$ & 79 & white \\
\hline 5 & $4-\mathrm{N}\left(\mathrm{CH}_{3}\right)_{2}$ & $222-224$ & 83 & orange \\
\hline 6 & $\mathrm{H}$ & $156-158$ & 89 & white \\
\hline 7 & $4-\mathrm{No}_{2}$ & $136-138$ & 77 & Pale yellow \\
\hline 8 & $2-\mathrm{OMe}$ & $166-168$ & 80 & orange \\
\hline 9 & $4-\mathrm{OMe}$ & $151-153$ & 84 & white \\
\hline 10 & 2-CL & $136-138$ & 75 & Pale yellow \\
\hline
\end{tabular}


Synthesis of some azetidonone and 1, 3-oxazepine deritives from thymol.

Table (2): Some physical constant for compound (11-16)

\begin{tabular}{|c|c|c|c|c|}
\hline Comp. No. & R & M.p. C & Yield (\%) & Color \\
\hline 11 & $4-\mathrm{CL}$ & $126-128$ & 59 & brown \\
\hline 12 & $4-\left(\mathrm{NCH}_{3}\right)_{2}$ & $142-144$ & 67 & Pale brown \\
\hline 13 & $\mathrm{H}$ & $120-122$ & 75 & Pale brown \\
\hline 14 & $4-\mathrm{NO}_{2}$ & $148-150$ & 79 & Pale brown \\
\hline 15 & $2-\mathrm{OMe}$ & $143-145$ & 57 & white \\
\hline 16 & $4-\mathrm{OMe}$ & $137-139$ & 58 & white \\
\hline
\end{tabular}

Table (3): Some physical constant for compounds (17-22)

\begin{tabular}{|c|c|c|c|c|}
\hline Comp. No. & $\mathrm{R}$ & $\mathrm{Mp}\left(\mathrm{C}^{\circ}\right)$ & Yield (\%) & Color \\
\hline 17 & $4-\mathrm{OH}$ & $115-117$ & 73 & yellow \\
\hline 18 & $4-\mathrm{CL}$ & $173-174$ & 84 & white \\
\hline 19 & $4-\left(\mathrm{NH}_{3}\right)_{2}$ & $103-107$ & 75 & red \\
\hline 20 & $\mathrm{H}$ & $148-150$ & 69 & white \\
\hline 21 & $4-\mathrm{No}_{2}$ & $151-153$ & 53 & yellow \\
\hline 22 & $4-\mathrm{CL}$ & $180-181$ & 75 & white \\
\hline
\end{tabular}<smiles>CCOC(=O)C(C)(C)C(=O)Oc1cc(C)ccc1C(C)C</smiles><smiles>CCOC(=O)COc1cc(C)ccc1C(C)C</smiles>

(1)<smiles>Cc1cc(NN)c(C(C)C)c(OCC(N)=O)c1</smiles>

(2)

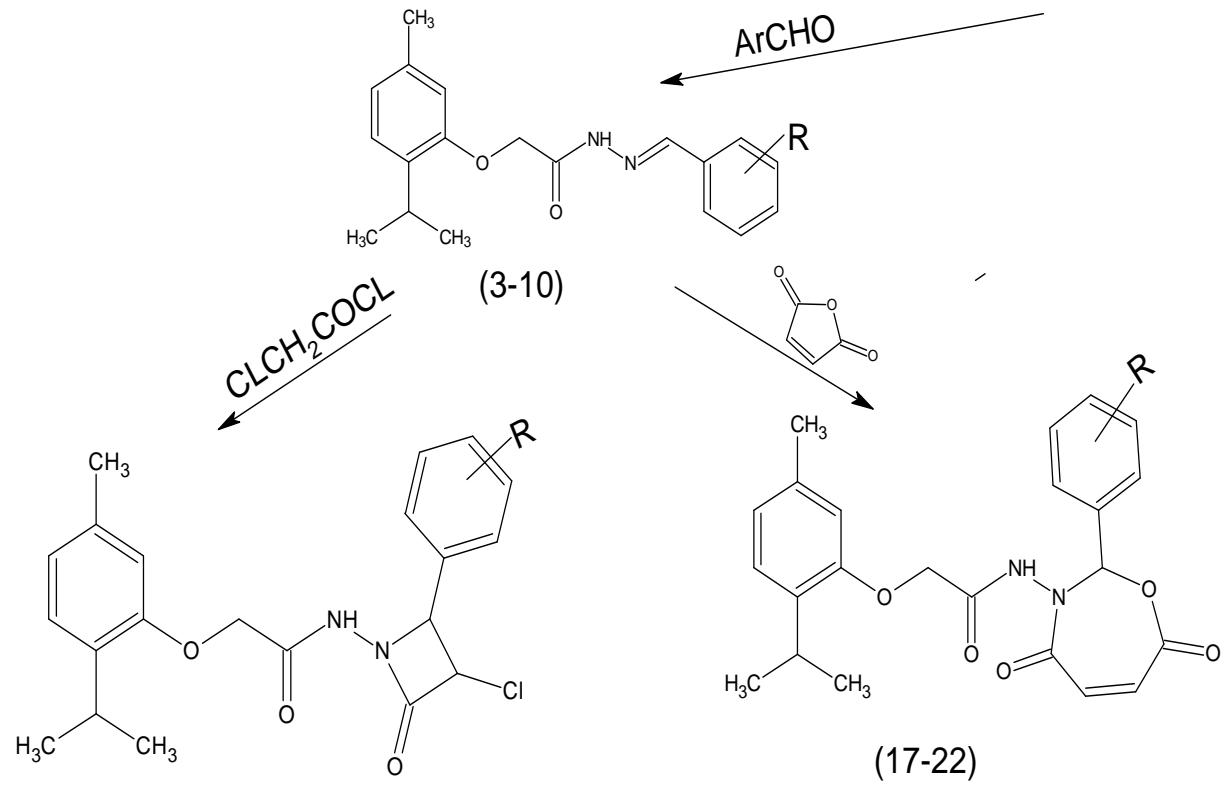

$(11-16)$ 
Table (4): Spectral data for hydrazones (3-10)

\begin{tabular}{|c|c|c|c|c|c|}
\hline $\begin{array}{c}\text { Comp. } \\
\text { NO. }\end{array}$ & $\mathrm{R}$ & \multicolumn{4}{|c|}{$\mathrm{IRrcm}^{-1}(\mathrm{KBr})$} \\
\cline { 3 - 6 } & & $\mathrm{N}-\mathrm{H}$ & $\mathrm{C}=\mathrm{O}$ & $\mathrm{C}=\mathrm{N}$ & others \\
\hline 3 & $4-\mathrm{O}-\mathrm{H}$ & 3317 & 1673 & 1614 & $3071(\mathrm{O}-\mathrm{H})$ \\
\hline 4 & $4-\mathrm{CL}$ & 3444 & 1685 & 1613 & $726(\mathrm{C}-\mathrm{CL})$ \\
\hline 5 & $4-\mathrm{N}\left(\mathrm{CH}_{3}\right)_{2}$ & 3451 & 1692 & 1612 & $1257(\mathrm{C}-\mathrm{N})$ \\
\hline 6 & $\mathrm{H}$ & 3444 & 1670 & 1604 & \\
\hline 7 & $4-\mathrm{NO}_{2}$ & 3443 & 1687 & 1613 & $\left.\begin{array}{c}\left.1284 \text { sy.(NO } \mathrm{NO}_{2}\right) \\
1506 \text { as.(NO }\end{array}\right)$ \\
\hline 8 & $2-\mathrm{OMe}$ & 3446 & 1697 & 1606 & $\begin{array}{c}1104 \text { sy.(C-O-C) } \\
1256 \text { ay.(C-O-C) }\end{array}$ \\
\hline 9 & $4-\mathrm{OMe}$ & 3487 & 1680 & 1612 & $\begin{array}{c}1101 \text { sy.(C-O-C) } \\
1242 \text { ay.(C-O-C) }\end{array}$ \\
\hline 10 & $2-\mathrm{CL}$ & 3445 & 1688 & 1604 & $758(\mathrm{C}-\mathrm{CL})$ \\
\hline
\end{tabular}

Table (5): Spectral data for substituted azetidin-2-one derivative (11-16)

\begin{tabular}{|c|c|c|c|c|c|c|c|c|}
\hline \multirow{2}{*}{$\begin{array}{l}\text { Comp. } \\
\text { No. }\end{array}$} & \multirow{2}{*}{\multicolumn{2}{|c|}{$\mathrm{R}$}} & \multicolumn{6}{|c|}{$\mathrm{IRr} \mathrm{cm}^{-1}(\mathrm{KBr})$} \\
\hline & & & $\begin{array}{c}\mathrm{C}=\mathrm{O} \\
\text { Lactone }\end{array}$ & $\begin{array}{l}\mathrm{C}=\mathrm{O} \\
\text { amide }\end{array}$ & $\mathrm{C}-\mathrm{N}$ & $\mathrm{Arc}=\mathrm{c}$ & $\mathrm{C}-\mathrm{CL}$ & others \\
\hline 11 & \multicolumn{2}{|c|}{ 4-CL } & 1696 & 1647 & 1430 & 1504 & 762 & \\
\hline 12 & \multicolumn{2}{|c|}{$4-\mathrm{N}\left(\mathrm{CH}_{3}\right)_{2}$} & 1717 & 1652 & 1424 & 1507 & 711 & C-N(1245) \\
\hline 13 & \multicolumn{2}{|c|}{$\mathrm{H}$} & 1700 & 1613 & 1433 & 1505 & 754 & \\
\hline 14 & \multicolumn{2}{|c|}{$4-\mathrm{NO}_{2}$} & 1733 & 1687 & 1435 & 1508 & 749 & $\begin{array}{l}1315 \text { sy. }\left(\mathrm{NO}_{2}\right) \\
1435 \text { as. }\left(\mathrm{NO}_{2}\right)\end{array}$ \\
\hline 15 & \multicolumn{2}{|c|}{ 2-OMe } & 1717 & 1652 & 1422 & 1507 & 750 & $\begin{array}{l}1100 \text { sy.(C-O-C) } \\
1163 \text { ay.(C-O-C) }\end{array}$ \\
\hline 16 & \multicolumn{2}{|c|}{ 4-OMe } & 1717 & 1650 & 1425 & 1503 & 745 & $\begin{array}{l}1103 \text { sy.(C-O-C) } \\
1168 \text { as.(C-O-C) }\end{array}$ \\
\hline \multicolumn{3}{|c|}{ Comp.No. } & $\mathrm{R}$ & \multicolumn{5}{|c|}{$\begin{array}{l}{ }^{1} \mathrm{HNMR} \delta(\mathrm{ppm}) \\
\text { Solv.CDCL }\end{array}$} \\
\hline 16 & & \multicolumn{2}{|c|}{ 4-OMe } & \multicolumn{5}{|c|}{$\begin{array}{l}1.245(\mathrm{~m}, 6 \mathrm{H}) 2\left(\mathrm{CH}_{3}\right)_{2} \\
2.314(\mathrm{~s}, 3 \mathrm{H}) \mathrm{Ar}-\mathrm{CH}_{3} \\
3.272(\mathrm{~m}, 1 \mathrm{H}) \mathrm{CH} \\
3.838(\mathrm{~s}, 3 \mathrm{H}) \mathrm{OCH}_{3} \\
4.132(\mathrm{~s}, 2 \mathrm{H}) \mathrm{CH}_{2} \\
4.615(\mathrm{~d}, 1 \mathrm{H}) \mathrm{CHCL} \\
5134(\mathrm{~s}, 1 \mathrm{H}) \mathrm{CH}, \mathrm{cyclic} \\
6.602-8.137(\mathrm{~m}, 7 \mathrm{H}) \mathrm{Ar}-\mathrm{H} \\
\text { 9.049(s,1H)NH}\end{array}$} \\
\hline
\end{tabular}


Synthesis of some azetidonone and 1, 3-oxazepine deritives from thymol.

Table (6): Spectral data for compounds (17-22)

\begin{tabular}{|c|c|c|c|c|c|c|c|c|}
\hline \multirow{2}{*}{$\begin{array}{l}\text { Comp. } \\
\text { No. }\end{array}$} & \multirow[t]{2}{*}{$\mathrm{R}$} & \multicolumn{7}{|c|}{$\mathrm{IR} r \mathrm{Cm}^{-1}(\mathrm{KBr})$} \\
\hline & & $\begin{array}{l}\mathrm{C}=\mathrm{O} \\
\text { Lactone }\end{array}$ & $\begin{array}{l}\mathrm{C}=\mathrm{O} \\
\text { amide }\end{array}$ & $\mathrm{C}-\mathrm{N}$ & $\mathrm{C}-\mathrm{O}-\mathrm{C}$ & $\begin{array}{l}\mathrm{C}=\mathrm{C}- \\
\mathrm{C}=\mathrm{O}\end{array}$ & $\operatorname{Arc}=\mathrm{c}$ & others \\
\hline 17 & $4-\mathrm{OH}$ & 1681 & 1654 & 1417 & $\begin{array}{l}\text { sy/as } \\
1167 \\
1242\end{array}$ & 1611 & 1507 & \\
\hline 18 & 4-CL & 1693 & 1606 & 1413 & $\begin{array}{l}1168 \\
1253\end{array}$ & 1578 & 1504 & $\begin{array}{l}\text { C-CL } \\
739\end{array}$ \\
\hline 19 & $4-\mathrm{N}\left(\mathrm{CH}_{3}\right)$ & 1697 & 1613 & 1434 & $\begin{array}{l}1169 \\
1257\end{array}$ & 1540 & 1506 & $\begin{array}{l}\mathrm{C}-\mathrm{N} \\
1257\end{array}$ \\
\hline 20 & $\mathrm{H}$ & 1700 & 1612 & 1414 & $\begin{array}{l}1171 \\
1256 \\
\end{array}$ & 1581 & 1504 & \\
\hline 21 & $4-\mathrm{NO}_{2}$ & 1689 & 1505 & 1410 & $\begin{array}{l}1168 \\
1254\end{array}$ & 1586 & 1504 & $\begin{array}{l}\mathrm{NO}_{2} \mathrm{~S} \\
\text { ylas } \\
1339 \\
1379\end{array}$ \\
\hline 22 & $2-\mathrm{CL}$ & 1693 & 1603 & 1420 & $\begin{array}{l}1170 \\
1261 \\
\end{array}$ & 1577 & 1506 & $\begin{array}{l}\mathrm{C}-\mathrm{CL} \\
749 \\
\end{array}$ \\
\hline Comp.No. & $\mathrm{R}$ & \multicolumn{7}{|c|}{$\begin{array}{l}{ }^{1} \mathrm{HNMR} \delta(\mathrm{ppm}) \\
\text { Solv.CDCL }_{3}\end{array}$} \\
\hline 18 & 4-CL & \multicolumn{7}{|c|}{$\begin{array}{l}1.286(\mathrm{~m}, 6 \mathrm{H}) 2\left(\mathrm{CH}_{3}\right)_{2} \\
2.303(\mathrm{~s}, 3 \mathrm{H}) \mathrm{Ar}-\mathrm{CH}_{3} \\
3.289(\mathrm{~m}, 1 \mathrm{H}) \mathrm{CH} \\
4.648(\mathrm{~s}, 2 \mathrm{H}) \mathrm{O}-\mathrm{CH}_{2-}- \\
5.118(\mathrm{~s}, 1 \mathrm{H})-\mathrm{N}-\mathrm{CH}-\mathrm{O} \\
6.458(\mathrm{~d}, 1 \mathrm{H}) \mathrm{CH}=\mathrm{COO} \\
6.636-7.694(\mathrm{~m}, 7 \mathrm{H}) \mathrm{Ar}-\mathrm{H} \\
7.721(\mathrm{~d}, 1 \mathrm{H}) \mathrm{CHCO} \\
9.355(\mathrm{~s}, 1 \mathrm{H}) \mathrm{NH}\end{array}$} \\
\hline
\end{tabular}




\section{References}

1) A. Rajasekaran, M. Periasamy and S. Venkatesan, J. Dev. Biol. Tissu Eng.Vol.2(1), 5, (2010).

2) P.Shanmugapandiyan, K.S. Denshing, R. IIavarasan, N. Anbalagan, R.Nirmal, International Journal of Sciences and Drug Research, 2(2), 115, (2010).

3) S.Jubie, B.Gowramma, N.K.Muthal, R.Kalirajan, S.Gomahi and Kelang, International Journal of chem. Tech Research. Vol.1, No.2. P153 (2009).

4) BG. Mohmed, AA. Abdel-Alim, MA. Hussein, Acta Pharm. 56(1), 31 (2005)- Hue, B.Palomba, M.Giacordy-pety-Botti, R-ALric and P.Prit, Ther.drug Man It., 20,335 (1998).

5) J.R. Moody, D.Zhang, T.M.Heize and C.E. Cerniglia. Applied and Environmental Microbiology, 66(8), 3646 (2000).

6) G.S.Singh and P.Luntha, Contents, European, J.Med. Chem. 44, 2269, (2009).

7) I.K.Bhat, S.K. Chaithanya, P.D. Satyanarayana, B. Kalluraya, J.Serb. Chem. Soc. 72(5), 437(2007).

8) N.Khan, A.Razzaq, Z.Baber, S.Alamm, J.Saudi chem. Soc., Vol.4, No.1,109 (2000).

9) B.S. Vashi, D.S. Mehta and V.H. Sheb, Indian Journal of Chemistry, 35B, 111(1996).

10) A.N, Ali, Ph.D. Thesis, University of Mosul, Mosul-Iraq, (2006)

11) M.P. Toraskar, V.J. Kulkarni., Chem. Tech. Res. Vol.4, No.4, 1194 (2009).

12) R.M. Silvertein, G.C. Bassler and T.C. Morrill, 'Spectrometric identification of Organic Compounds' 3rded., John Wiley and Sons, Inc, New York,100, (1974). 